\title{
Diagnosis of heart valve stenosis through the use of artificial neural networks
}

\author{
S. Kara ${ }^{1}$, A. Güven ${ }^{2}$, M. Okandan ${ }^{1} \&$ F. Dirgenali ${ }^{1}$ \\ 'Dept. of Electronics Engineering, Erciyes University, Turkey. \\ ${ }^{2}$ Civil Aviation School, Erciyes University, Turkey.
}

\begin{abstract}
One of the current practices of diagnosing valve stenosis is based on the investigation of the Doppler Ultrasound's Fast Fourier Transformation (FFT) sonogram. This method depends on the physicians' interpretation and experience and sometimes results in false diagnosis. In this study, we have facilitated Artificial Neural Networks (ANN) that will not only simplify the diagnosis but enable the physician to make a quicker judgment about the existence of stenosis, without any hesitation. The FFT sonogram of Doppler Heart Ultrasound of a healthy person very resembles to the "M" letter. The ratios of the three points (First Systolic Peak, Endpoint of Diastole, Second Systolic Peak) in the M like curve are used as inputs to our ANN system, which is trained using Levenberg-Marquardt Method. Our system is tested on 20 patients, who were subjected for recording of the Doppler Ultrasound at mitral valves. Sixteen patients' data is allocated for training purposes and four patients were tried to see whether the ANN trained complies with physicians direct diagnosis from the FFT sonogram. The testing results was found to be compliant with physicians' findings regarding the existence and level of stenosis. The fuzzy appearance of the sonogram sometimes makes physicians suspicious about the existence of stenosis. Our technique gets around this problem by using ANN to decide and assists the physician to make the final judgment in confidence. The particular hallmark of this system is being an Add-on solution which can be coupled to current already installed Doppler ultrasounds.
\end{abstract}

\section{Introduction}

Electrocardiography is one of the early techniques developed to diagnose heart disorders. However, especially in the diagnosis of heart valve stenosis, ECG is not 


\section{4}

Simulations in Biomedicine $V$

competent enough. For such cases, Doppler Sonograms derived from the blood flow through the valves are preferred, instead. Doppler shift frequency which is directly proportional to the blood flow speed is subjected to spectral analysis that yield the sonograms showing the change of the Doppler spectrum with respect to time. This analysis enables the physicians to gather very important clinical information about the cardiovascular health of the patient. In order to produce the sonograms, the Doppler signals are sampled at appropriate frequencies and then grouped in certain number of data points. Afterwards, FFT is applied to each group in order to obtain their power spectral density functions. When these power spectral density functions are sequenced and plotted on the time line, they present the change of Doppler Signal Sonogram Graphics. The envelope of this Sonogram Plot, which resembles to the " $\mathrm{M}$ " letter, is facilitated particularly for diagnosing heart valve stenosis [1].

Diagnosing stenosis through the investigation of the Doppler Ultrasound's FFT sonogram is imperfect and requires some additional work. Because, the final diagnosis depends on the physicians' interpretation and experience and this sometimes results in false diagnosis. In this study, we have facilitated Artificial Neural Networks (ANN) that will not only simplify the diagnosis but also enable the physician to make a quicker judgment about the existence of stenosis, without any hesitation.

In this research, we worked on the utilization of ANN system that we built on the Doppler Signals' FFT Sonogram. The most important thing about ANN is that it works as an expert system which will eventually help the physicians with the decision making process about the existence of the stenosis. ANN is trained with the available data samples to explore the relation between inputs and outputs so that you can reach the proper outputs when you enter some new input data $[1,2,3]$.

\section{Materials and methods}

The ultrasound transducer that works in the range of $2-3,25 \mathrm{MHz}$ is located on the apex region of the patients and focused to the mitral valve to receive the appropriate signals, which will be processed later to obtain the Doppler shift frequencies. Doppler shift frequency signals are captured through the audio output of the ultrasound machine. The received signals are subjected to the 8-bit Analog to Digital conversion and then transferred to a $\mathrm{PC}$ via an $\mathrm{I} / \mathrm{O}$ card $[4,5]$. FFT is applied to these received Doppler Shift signals.

The FFT sonogram of Doppler Hearth Ultrasound of a healthy person very resembles to the "M" letter. The ratios of the three points (1- First Systolic Peak, 2Endpoint of Diastole 3-Second Systolic Peak) in the M like curve are used as inputs to our ANN system $[6,7,8,9,10]$.

A multilayered perceptron (MLP), which makes up the network structure, consists of three types of layers: input layer, output layer and one or more hidden layers. Each neuron in the layer consists of two parts: the net function and the activation function. The net function determines how the network inputs are combined inside the neuron. In this sense, the weighted linear summation is adapted as the net function. The output of the neuron is related to the network input via a linear or nonlinear transformation called the activation function. In various neural 
network models, different activation functions have been proposed $[3,11,12,13]$. In our MLP, three neurons are employed at the input layer while four neurons are used at the output layer. Linear transfer function is used both at the input and output layers as the activation function.

In the first and second hidden layer, there are five and six neurons respectively. Tangential sigmoid transfer function is applied at these two hidden layers as the activation function of each neuron.

MLPs can be trained using many different learning algorithms. We have chosen Levenberg Marquart learning algorithms to train the MLP. Low error rate and fast result convergence are main reasons that we have chosen this method $[11,12,13]$.

\section{Results}

First Systolic Peak (FSP), Endpoint of Diastole (EOD) and Second Systolic Peak $(\boldsymbol{S S P})$, in the M like curve of the FFT Sonograms of healthy persons and patients with different level of stenosis at their mitral valves make up the basis for input data set. The ratios of these three data points (seen in Table 1) are used as inputs to the training and testing sessions. Sixteen patients' data is allocated for training purposes and four patients were tried to see whether the ANN trained complies with physicians direct diagnosis from the FFT sonogram.

After many iterations, it was found that the most suitable structure with the least error of $3,6262.10^{-6}$ mean square error (at 31 Epoch) was achieved when five and six neurons are used at the two hidden layers. The variation of system error rate with respect to the epoch number during training iterations is shown in Figure 1. As seen in this figure, the results are stable and no fluctuations are observed. This indicates that the selected parameters are the most proper set for a minimum error rate. As a part of checking the built ANN, four of the patients' data were subjected to testing. The end results are classified in four different groups as Healthy Person, Low-level of Stenosis, Medium-level of Stenosis and High-level of stenosis. Testing results were found to be compliant with the physician's diagnosis directly from the FFT sonogram.

\section{Discussion and Conclusion}

The stenosis at the mitral valves are investigated by recording and studying the Doppler frequency shift signals obtained from the apex region. True and accurate interpretation of FFT sonogram analysis of these signals is very crucial in diagnosing disorders with the heart valves. However, it requires some real experience to conclude reliable information about the health of the valves. The fuzzy appearance of the sonogram sometimes makes physicians suspicious about the existence of stenosis and causes false diagnosis. Our technique gets around this problem by using ANN to decide and assists the physician to make the final judgment in confidence. The particular hallmark of this system is being an Add-on solution, which can be coupled to current already, installed Doppler ultrasounds for an economical value. This study had very successful results when physicians` 
Table 1 Input and Output Values for the training and testing of the ANN

a) Input Values for the training session

\begin{tabular}{|c|l|l|l|}
\hline $\begin{array}{c}\text { Patient } \\
\text { No }\end{array}$ & $\begin{array}{c}\text { FSP } / \\
\text { EOD }\end{array}$ & $\begin{array}{l}\text { SSP/ } \\
\text { EOD }\end{array}$ & $\begin{array}{c}\text { FSP/ } \\
\text { SSP }\end{array}$ \\
\hline 1 & 3.7 & 1.88 & 1.97 \\
\hline 2 & 3.0 & 1.5 & 2.0 \\
\hline 3 & 2.08 & 1.48 & 1.403 \\
\hline 5 & 2.0 & 1.7 & 1.176 \\
\hline 6 & 2.5 & 2.37 & 1.052 \\
\hline 7 & 2.2 & 1.83 & 1.22 \\
\hline 8 & 2.09 & 1.63 & 1.277 \\
\hline 10 & 1.9 & 1.76 & 1.074 \\
\hline 11 & 1.66 & 1.52 & 1.092 \\
\hline 12 & 1.96 & 1.78 & 1.095 \\
\hline 14 & 1.9 & 1.43 & 1.333 \\
\hline 15 & 1.9 & 0.58 & 3.285 \\
\hline 16 & 1.52 & 1.16 & 1.31 \\
\hline 17 & 1.6 & 1.18 & 1.34 \\
\hline 18 & 2.2 & 0.39 & 5.666 \\
\hline 19 & 1.5 & 1.66 & 0.9 \\
\hline
\end{tabular}

c) Input Values for testing session

\begin{tabular}{|c|c|l|l|}
\hline $\begin{array}{c}\text { Patient } \\
\text { No }\end{array}$ & $\begin{array}{c}\text { FSP/ } \\
\text { EOD }\end{array}$ & $\begin{array}{c}\text { SSP/ } \\
\text { EOD }\end{array}$ & $\begin{array}{c}\text { FSP/ } \\
\text { SSP }\end{array}$ \\
\hline 4 & 3.25 & 1.8 & 1.805 \\
\hline 9 & 2.39 & 2.1 & 1.134 \\
\hline 13 & 1.79 & 1.65 & 1.086 \\
\hline 20 & 2.28 & 0.66 & 3.42 \\
\hline
\end{tabular}

b) Output Values entered for the training session

\begin{tabular}{|c|c|c|c|c|}
\hline $\begin{array}{c}\text { Patient } \\
\text { No }\end{array}$ & $\begin{array}{c}\text { Healthy } \\
\text { Person }\end{array}$ & $\begin{array}{c}\text { Low } \\
\text { Stenosis }\end{array}$ & $\begin{array}{c}\text { Medium } \\
\text { Stenosis }\end{array}$ & $\begin{array}{c}\text { High } \\
\text { Stenosis }\end{array}$ \\
\hline 1 & 1 & 0 & 0 & 0 \\
\hline 2 & 1 & 0 & 0 & 0 \\
\hline 3 & 1 & 0 & 0 & 0 \\
\hline 5 & 0 & 1 & 0 & 0 \\
\hline 6 & 0 & 1 & 0 & 0 \\
\hline 7 & 0 & 1 & 0 & 0 \\
\hline 8 & 0 & 1 & 0 & 0 \\
\hline 10 & 0 & 0 & 1 & 0 \\
\hline 11 & 0 & 0 & 1 & 0 \\
\hline 12 & 0 & 0 & 1 & 0 \\
\hline 14 & 0 & 0 & 0 & 1 \\
\hline 15 & 0 & 0 & 0 & 1 \\
\hline 16 & 0 & 0 & 0 & 1 \\
\hline 17 & 0 & 0 & 0 & 1 \\
\hline 18 & 0 & 0 & 0 & 1 \\
\hline 19 & 0 & 0 & 0 & 1 \\
\hline
\end{tabular}

d) Output values obtained at the testing session

\begin{tabular}{|c|c|c|c|c|}
\hline $\begin{array}{c}\text { Patient } \\
\text { No }\end{array}$ & $\begin{array}{c}\text { Healthy } \\
\text { Person }\end{array}$ & $\begin{array}{c}\text { Low } \\
\text { Stenosis }\end{array}$ & $\begin{array}{c}\text { Medium } \\
\text { Stenosis }\end{array}$ & $\begin{array}{c}\text { High } \\
\text { Stenosis }\end{array}$ \\
\hline 4 & 1 & 0 & 0 & 0 \\
\hline 9 & 0 & 1 & 0 & 0 \\
\hline 13 & 0 & 0 & 1 & 0 \\
\hline 20 & 0 & 0 & 0 & 1 \\
\hline
\end{tabular}



Figure 1: Variation of the error rate with respect to the epoch number in the ANN 
diagnosis directly through FFT sonogram analysis was compared with our testing results. As the next step of the research, we will include the study of tricuspid valve stenosis and try other methods to train the ANN in order to reach the possible least error rate.

\section{References}

[1] Evans, D.H., Skidmore, W. N., Doppler Ultrasound: Physics, Instrumentation and Clinical Applications, John Wiley \& Sons Ltd., 1989.

[2] Simpson, P.K., Artificial Neural Systems, Pergamon Press, 1989.

[3] Chen, C. H., Fuzzy Logic and Neural Network Handbook, McGraw-Hill, Inc., 1996.

[4] Kara, S., A Study of Mitral and Tricuspid Valve Blood Flows by Autoregressive Spectral Analysis Method and Doppler Unit, Ph. D. Thesis, Erciyes University, 1994.

[5] Kara, S., Ertaş, G., Computer Supported Pourcelot's Resistance Index and S/D Ratio Measurement of Blood Flow, $2^{\text {nd }}$ International Biomedical Engineering Days, pp. 23-25, 1998.

[6] Dokur, Z., Ölmez, T., Yazgan, E., Comparison of Discrete Wavelet and Fourier Transforms for ECG Beat Classification, Electronics Letters, Vol. 35, No 18, 1502-1504, 1999.

[7] Dokur, Z., Ölmez, T., Korürek, M., Yazgan, E., Detection of ECG Waveforms By Using Artifical Neural Networks, Proc. of the $18^{\text {th }}$ IEEE-EMBS Annual Int. Conference, Nov. 1996.

[8] Duda, R.O. and Hart, P.E., Pattern Classification and Scene Analysis, Stanford Research Institute, 1989.

[9] Baykal, N., Reggia, J.A., Yalabık, N., Erkmen, A., Beksac, M.S., Feature Discovery and Classification of Doppler Umbilical Artery Blood Flow Velocity Waveforms, Computing in Medicine \& Biology, 26(6), pp. 451-462, 1996.

[10] Hagan, M.T., Menhaj, M., Trainin Feedforward Networks with the Marquart Algorithm, IEEE Transactions on Neural Networks, 5(6), pp. 989-993, 1994.

[11] Maren, A., Harston C. and Pap, R., Handbook of Neural Computing Applications, Academic Press, London, ISBN 0-12-471260-6, 1990.

[12] Rubelhart, D.E., McClelland, J.L., Parallel Distributed Processing, Vol. 1, MIT Press, Cambridge, 1986.

[13] Hagan, M. T., Demuth, H. B. and Beale, M. H., Neural Network Design, Ma: Pws Publishing, Boston, 1996. 
